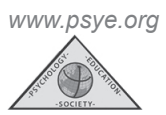

\title{
Claves y evidencias del uso de las TIC en trastorno mental grave
}

\author{
Juana Mªteu-Mateu* y Noelia Navarro-Gómez \\ Universidad de Almería, España
}

(Recibido 12 Diciembre, 2014; Aceptado 25 Enero, 2015)

RESUMEN: El modo de organizarse y relacionarse ha sufrido profundos cambios desde la irrupción de las tecnologías de la información y la comunicación (TIC). La presencia de éstas es cada vez más frecuente y necesaria. La evaluación de las consecuencias del uso de las TIC es importante a nivel general, pero más aún en poblaciones específicas como en las personas diagnosticadas con trastorno mental grave (TMG). Por este motivo, el objetivo de este estudio es analizar las claves y evidencias presentes en la literatura acerca de la aplicación y el uso de las TIC en personas con TMG, así como los beneficios y/o perjuicios que pudieran derivarse. El análisis abarca el uso de las TIC como herramienta de búsqueda de información, para la participación en grupos de apoyo online y para el establecimiento de relaciones sociales. Los resultados a nivel general indican las ventajas de las TIC para la integración en la sociedad e incluso para la mejora clínica en personas con TMG. Sin embargo, variables como la poca alfabetización digital, la ausencia de una buena relación terapéutica y/o ciertas características de las TIC, pueden mermar los efectos positivos.

Palabras clave: tecnología de la información y la comunicación, esquizofrenia, alfabetización digital e internet.

\section{Keys and evidences of the use of ICT in severe mental disorder}

\footnotetext{
ABSTRACT: The way of organizing and relating has suffered deep change since the emergence of the information and communica tiontechnologies (ICT). Their presenceis more and more common and necessary. In general, the evaluation of the consequences of the use of ICT is important but it is even more in specific population such as peoplediagnosedwithsevere mental disorder (SMD). For this reason, the objetive of this study is to analyze the information reported in the literature about the keys and evidences of the application and use of ICT in people with SMD, as well as the benefits and damages of them. The analysis includes the use of ICT as search tool, for participation in online support groups and for the development of social relationships. In general, the results show the advantages of ICT for social integration and clinical improvement of the people with SMD. Nevertheless, some variables can reduce the positive effects such as the little digital literacy, the lack of therapy relation and several features of ICT. Keywords: information and communication technologies, schizophrenia, digital literacy and internet. 


\section{INTRODUCCIÓN}

Desde la irrupción a finales del siglo XX y principios del XXI de las Tecnologías de la Información y la Comunicación (TIC) en la sociedad, estos recursos han ido adquiriendo un papel más recurrente y relevante en nuestro día a día. Como es bien sabido, el surgimiento de importantes innovaciones tecnológicas ha implicado cambios en la estructura económica, social y laboral, así como en el comportamiento habitual de las personas (Gergen, 1992). La aplicación de las TIC en la sociedad moderna del s.XXI ha facilitado el avance y la expansión de numerosas actividades como el comercio, la ciencia, la educación o la comunicación a distancia, entre otras. La relevancia de éstas es tal, que el nivel de acceso que un país tiene a las TIC es considerado un indicador de desarrollo por el Banco Mundial de Datos (World Bank Institute, 2008). Uno de los datos que muestra la importante presencia de las TIC en España es el aumento en diez años de los usuarios que se conectaron, al menos una vez a Internet desde cualquier tipo de dispositivo, siendo el $39.9 \%$ de la población en el año 2003 y el 71,6\% en el año 2013 (World Bank Institute, 2015). El aumento del uso de esta TIC junto a otras se ha producido en la población general debido al fácil acceso, el abaratamiento y la existencia de un amplio catálogo de dispositivos que se ajustan a las necesidades del consumidor.

A lo largo de la literatura científica se puede observar el interés por estudiar las consecuencias que a nivel psicológico pueden tener el uso de las TIC. A nivel general, se ha analizado la influencia sobre aspectos del autoconocimiento como por ejemplo, la identidad del "Yo" (p.ej., García Montes, Caballero Muñoz y Pérez Álvarez, 2006) o la autoestima (p.ej., Gonzales y Hancock, 2010) entre otros. Además, se ha investigado los efectos sobre las prácticas sociales y las relaciones interpersonales (p.ej., AmichaiHamburguer y Hayat, 2011; Cáceres, Ruiz y Brändle, 2009; García y Del Hoyo, 2013). Entre estos tipos de estudios se hallan los que tienen como objetivo analizar el uso de las TIC en poblaciones específicas ya sea en poblaciones con problemas de salud física (p.ej., Abt, Pablo, Serrano, Fernández y Martín, 2013; Greene, Niteesh, Choudhry, Kilabuk, Shrank, 2010) o en poblaciones con algún trastorno psicológico (p.ej., Buffardi y Campbell, 2008; Haker, Lauber y Rössler, 2005; Vázquez y Martínez, 2011). Éstos últimos, suelen centrarse en analizar los beneficios y/o perjuicios que el uso de las TIC puede tener para estas poblaciones. Una revisión de la literatura sobre el uso de las TIC en personas diagnosticadas con trastorno mental grave (TMG), indica un interés reciente por la temática, en correspondencia con el aumento de personas con TMG que han incorporado a sus vidas las TIC. Sin embargo, a nivel general la investigación sobre las TIC es aún escasa (Kaplan, Salzer, Solomon, Brusilovskiy y Cousounis, 2011), y suele indicar cierta controversia acerca de las ventajas y las desventajas que el uso de las TIC pueden tener en esta población. No obstante, en los últimos años, se ha dado un mayor número de estudios en favor a que tienen más beneficios (p.ej. Haker et al., 2005; Ritterband et al., 2003; Veretilo y Billick, 2012) que perjuicios (p.ej. Nitzan, Shoshan, Lev-Ran y 
Fenning, 2011).

El presente estudio tiene como objetivo hacer un análisis de las claves y evidencias presentes en la literatura científica acerca de la aplicación y el uso de las TIC en personas con TMG, así como los beneficios y/o perjuicios que pudieran derivarse. Para ello, se analizarán el uso de las TIC como instrumento para la obtención de la información, el acceso a grupos de apoyo a través de foros, chats y webs, y el uso de las redes sociales en TMG.

\section{Las TIC como instrumento para la búsqueda de información sobre TMG}

El fácil acceso y la obtención inmediata de la información requerida hacen que Internet sea una de las herramientas principales para la búsqueda de cualquier tipo de información. El interés por este instrumento como medio de obtención de información clínica es cada vez más frecuente en la sociedad, observándose tendencias distintas entre los diversos grupos poblacionales (Jiménez, García, Martín y Bermúdez, 2007). Frecuentemente, suelen ser personas adultas (Jiménez et al., 2007) con necesidad de resolver inmediatamente una duda o completar una información dada por el profesional sanitario. Además, las personas con una enfermedad mental o un trastorno psíquico que por su diagnóstico pueden ser estigmatizadas, serán las que más usen este medio (Berger, Wagner y Baker, 2005; Goldstein, 2002). De este modo, se refugian en el anonimato que ofrece internet y evitan ir a organismos públicos o centros sanitarios en los que puedan ser vistos y consecuentemente estigmatizados.

Las personas con TMG usuarias de internet reconocen haber buscado información sobre su trastorno o temas relacionados con él. La introducción de este recurso en la vida de las personas con TMG para la obtención de más información sobre el trastorno ha dado lugar a estudios en los que se investigan los recursos online utilizados (p.ej., Haker et al., 2005; Kaplan et al., 2011; Mazanderani, O’Neill y Powell, 2013; Schrank, Sibitz, Unger y Amering, 2010), así como la calidad de la información de los sitios webs (p.ej., Gorczynski, Patel y Ganguli, 2013; Read, 2008). Respecto a los recursos online utilizados, lo más frecuente es el acceso a la información a través de las páginas webs obtenidas como resultado del uso de un buscador y/o la participación en comunidades virtuales (p. ej., foros y chats) específicas para personas con TMG o bien problemas de salud mental.

Schrank et al., (2010) realizaron un estudio cualitativo a 26 personas con TMG, los cuáles informaron que el principal tema buscado en Internet fue sobre medicación, concretamente los efectos secundarios, así como la existencia de nuevos medicamentos que pudieran tener menos efectos secundarios. El siguiente tema más buscado hacía referencia al diagnóstico y la génesis del trastorno. La necesidad de buscar estas temáticas en Internet, además del miedo a ser estigmatizados, se corresponde con las quejas de desconocimiento acerca del trastorno debido a la información insuficiente aportada por los profesionales de salud mental y/o la ausencia de respuestas a las cuestiones planteadas en consulta (Guada y Venable, 2011; Dixon, Lucksted, Medoff, Burland y Stewart, 2011; Schrank et 
al., 2010).

Diversos autores (p.ej., Berger et al., 2005; Schrank et al., 2010); indican que la calidad de la información recibida suele ser sobreestimada por las personas con TMG. Estas personas suelen manifestar cierto temor de comentar la búsqueda de la información a su sanitario por si éste lo ve como una ofensa a su labor terapéutica. Sin embargo, este miedo no está presente cuando hay una buena relación terapéutica o bien el profesional respondió con naturalidad la primera vez que fue preguntado.

La preocupación porque Internet sea la única herramienta de obtención de información sobre salud ha llevado al interés por analizar la calidad de la información de las páginas webs, ya que si esta información es errónea o incompleta podría suponer una barrera para la adherencia al tratamiento o incluso para el establecimiento de la relación terapéutica. Read (2008) analizó la información contenida en 50 páginas webs y la comparó en relación a si la web era financiada por una empresa farmacéutica o por una empresa ajena a ésta. Los resultados de este estudio indicaron que la información aportada en la mayoría de los casos era incompleta. Además, las financiadas por la industria farmacéutica tendían a sesgar la información a favor de sus intereses económicos. Por ejemplo, las webs de compañías farmacéuticas fueron significativamente más propensas a explicar la génesis del trastorno a través de factores biogenéticos, omitiendo en muchos casos los factores psicosociales. Al igual que omitían la eficacia de las terapias psicológicas demostrada por la literatura (p.ej., Bach y Hayes, 2002; Gaudiano y Herbert, 2006; Gould, Mueser, Bolton, Mays y Goff, 2001) y sobreestimaban la eficacia de los medicamentos antipsicóticos sin mencionar los resultados negativos para ciertos síntomas o la tasa de abandonos debido a los efectos secundarios ya conocidos (Gónzalez y Pérez, 2007; Lieberman et al., 2005; Ross y Read, 2004 citado en Read, 2008). Akram, Boyter y Thompson (2010) en las 33 webs analizadas sobre medicación antipsicótica también hallaron información incompleta, al igual que les sucedieron a Gorczynski, Patel y Gaguli (2013) en el análisis de información sobre la actividad física y el deporte para personas con TMG.

Puesto que el uso de Internet es una realidad cada vez más presente, los profesionales deberían tenerlo en cuenta, dando más explicaciones sobre el trastorno y la confianza al paciente para preguntar sobre él. De esta manera, podrían contrastar la veracidad de la información obtenida a través de medios electrónicos. A su vez, debido a los buenos resultados obtenidos por las intervenciones psicoeducativas (p.ej., Dixon et al., 2004; Vigod et al., 2013) sería conveniente llevar a cabo en los organismos competentes programas psicoeducativos dirigidos tanto a las personas con el diagnóstico como a los familiares. Para aquellas personas cuya asistencia física al programa pueda suponer una barrera por el miedo al posible estigma o por la dificultad geográfica, la participación en un programa psicoeducativo online podría ser una solución.

Soto-Pérez y Franco-Martín (2014) desarrollaron un programa de intervención psicoeducativa (PsicoEd) para esquizofrenia administrado bajo dos modalidades, una presencial y otra online (los participantes de esta modalidad, tenían 
inconvenientes para acudir al presencial). Compararon la eficacia entre ambas modalidades y entre un grupo control. Los resultados obtenidos respecto al conocimiento del trastorno mostraron que los efectos de ambas modalidades fueron significativamente mayores en comparación al grupo control, sin embargo, entre ambas no hubo diferencias significativas aunque la presencial tuvo mejores resultados en el aprendizaje de conductas y la disminución de información errónea. A la vista de los resultados, podría decirse que los programas psicoeducativos online pueden ser una solución no sustitutiva de los programas presenciales pero sí especialmente útiles para cuando el acceso a éstos no es viable.

El acceso a foros o chats de autoayuda es otro medio por el que obtienen información al compartir el conocimiento de su trastorno con otras personas. Sin embargo, estos foros pueden desempeñar una función más social que informativa, pues permiten el intercambio de experiencias entre los usuarios lo que implica interacciones sociales online. Por este motivo, este tipo de TIC se va a tratar en el siguiente apartado.

\section{Grupos de apoyo/autoayuda para personas con TMG: foros, chats y webs}

Los foros o chats diseñados como grupos de apoyo o autoayuda principalmente tienen la función de permitir el intercambio de experiencias entre personas con alguna enfermedad concreta, cuidadores y/o familiares de enfermos, de tal manera que dicha experiencias sirvan de apoyo para personas con situaciones similares. La literatura sobre los beneficios de los grupos de apoyo en línea aún es escasa, aunque se muestran como una alternativa cuando hay dificultad para participar de forma presencial en grupos (Ritterband et al., 2003), o bien, la persona prefiere guardar su anonimato por miedo al estigma (Houston, Cooper y Ford, 2002).

Los resultados de los estudios sobre grupos de apoyo online para personas con TMG muestran que en la mayoría de las ocasiones pueden ser beneficiosos (Hakeret al., 2005., Kummervold, et al., 2001) salvo cuando hay una gran implicación emocional por parte del participante (Kaplan et al., 2010).

Principalmente, los foros suelen ser utilizados por esta población para la divulgación de experiencias personales y el intercambio de información sobre la medicación, los síntomas y las terapias psicológicas (Perron, 2002; Haker et al., 2005). Este intercambio de experiencias en muchos casos enriquece a los participantes aumentando su conocimiento e incluso teniendo esperanza cuando alguien expresa su mejoría.

Sin embargo, a diferencia de los resultados positivos que reporta una implicación emocional por parte de usuarios con otras enfermedades en los grupos de apoyo (p.ej., Houston et al., 2002; Winzelberg et al., 2003), en el caso de las personas con TMG no parece ser tan beneficiosa dicha implicación (Kaplan et al., 2010). En un ensayo controlado desarrollado por Kaplan et al., (2010) se diseñó un foro cumpliendo con las características habituales de éstos (no estructurados y no moderados). Se registraron medidas pre y post de bienestar psicológico y 
angustia, así como una entrevista sobre la experiencia en el foro. Los resultados indicaron que una mayor participación e implicación correlacionaba con una disminución del bienestar psicológico y mayores niveles de angustia, aunque los participantes informaron tener experiencias positivas al participar. No obstante, teniendo en cuenta que es una correlación, el aumento de la participación podría corresponder a periodos de sufrimiento (lo que explicaría los niveles obtenidos en angustia y bienestar psicológico) acudiendo el participante al foro como medida de apoyo.

En un estudio reciente realizado por Naslund, Grande, Aschbrenner y Elwyn (2014) se analizó en Youtube 19 vídeos de personas que se identificaron como personas con TMG y sus 3.044 comentarios correspondientes. De este análisis se desprende cuatro funciones que podrían desempeñar en los usuarios los vídeos y los comentarios: a) búsqueda de apoyo, b) disminuir la sensación de aislamiento y aumentar la de normalidad, c) compartir estrategias de afrontamiento y d) información sobre la experiencia con medicamentos y la atención sanitaria.Teniendo en cuenta la importante limitación de no hacer una evaluación clínica de cada participante y únicamente basarse en las verbalizaciones que éstos mostraban con sus comentarios, los autores indican que las funciones comentadas anteriormente parecen ser positivas para los usuarios.

El beneficio que los grupos de apoyo pueden dar a las personas con TMG parece evidente al igual que con el resto de poblaciones. No obstante, la importancia de que la implicación emocional pueda ser un hándicap ha de seguir investigándose a través de estudios controlados como el de Kaplan et al., (2010) pero evaluando qué función o funciones y consecuencias tienen para cada persona la participación en estos foros.

\section{Uso de las redes sociales en TMG}

En los últimos datos recogidos por el Instituto Nacional de Estadística (2014), se indica que más de la mitad de la población española $(51,1 \%)$ hace uso de las redes sociales. La comunicación online conlleva ciertas características tales como, la distorsión entre la percepción espacial y la distancia geográfica o la ausencia de elementos claves en la comunicación como es el lenguaje no verbal. Además, la comunicación a través de las redes sociales tiene el objetivo final de compartir con tu lista de contactos información personal, pudiéndose ver afectada la intimidad de la persona (Curtis, 2007) y más aún en aquellas que sean principiantes en el uso de éstas.

El interés principal de la literatura se ha centrado en investigar cómo las características de la comunicación online puede influir en el desarrollo de delirios (p. ej., Catalano, Catalano, Embi y Frankel, 1999; Compton, 2003) y si el uso de las redes sociales puede ser beneficioso para esta población. Hasta el momento, la literatura presente tiende a indicar que las redes sociales, en algunos casos, pueden tener efectos adversos para las personas con TMG (Krishna et al., 2010). Miller, Stewart, Schrimsher, Peeples y Buckley (2014) en su estudio sobre el 
uso de las TIC en TMG encontraron que, de 80 participantes, del 18\% al 36\% de los encuestados indicaban asociación entre el uso de las redes sociales y el empeoramiento de uno o varios síntomas psiquiátricos como la depresión o las alucinaciones. Además de la evidencia de los efectos adversos en personas diagnosticadas, el uso de las redes sociales o la comunicación online se ha relacionado con la aparición de sintomatología psicótica en personas sin antecedentes, como ocurrió en los tres casos presentados por Nitzan et al., (2011).

Un análisis de los casos presentados en la literatura que indican esos efectos negativos nos dirige a ciertas características de éstos que pueden ser influyentes para que esta herramienta no haya sido beneficiosa para ellos. El principal elemento presente en la mayoría de casos desfavorables es la escasa alfabetización digital. La ignorancia hacia el funcionamiento de la comunicación online puede dar lugar a malas interpretaciones en las interacciones con los demás o a un uso indeseado de la red social que a la larga puede favorecer a la formación de síntomas psicóticos. Otro elemento común en algunos casos es acudir a estas redes sociales creyendo que serán la solución al malestar psicológico.

Veretilo y Bates (2012) exponen un caso en el que la red social Facebook fue una ayuda para la recuperación de la persona, pues sirvió de medio para que volviera a establecer relaciones personales. Las claves de este caso se centran en que la persona tenía conocimientos previos del funcionamiento de este tipo de TIC y además, había una buena relación terapéutica con el terapeuta. El hecho de confiar en el terapeuta permitía que el paciente expresara sus preocupaciones, temores, etc., ante el uso de Facebook y, pese a tener ese temor, siguiera exponiéndose al contacto virtual con otras personas para posteriormente llevarlo a la vida real.

En Spinzy, Nitzan, Becker, Bloch y Fennig, (2012) se comparó el uso de las redes sociales entre población con síntomas psicóticos y población sin diagnóstico. Los resultados no indicaron diferencias a la hora de comunicarse a través de las redes sociales. Ambos grupos además de conocer a personas y comunicarse con ellas virtualmente, muchos establecieron contacto físico e incluso llegaron a ser personas determinantes en sus vidas.

Por lo tanto, el uso de la comunicación online, al igual que en la población general, puede tener beneficios siempre y cuando se use con conocimiento del funcionamiento de esta tecnología. De esta forma, se disminuiría la probabilidad de que ciertas características de esta comunicación puedan favorecer a la formación de delirios. Por otro lado, sería conveniente que los profesionales tuvieran en cuenta en sus evaluaciones la utilización de este medio, para informar (si fuera necesario) de las características de este tipo de comunicación, así como evaluar la posible utilidad para facilitar el establecimiento de relaciones sociales y su exposición en la vida real. 


\section{CONCLUSIÓN}

La implantación generalizada de las TIC en la sociedad está generando cambios en el modo de establecer relaciones sociales, laborales, y económicas. El buen uso de las TIC ha permitido que estos cambios sean en pros al desarrollo de la sociedad. La multitud de dispositivos y el aumento de la demanda han dado lugar a que el acceso a estas herramientas sea viable para muchas personas, lo que a su vez implica la generalización de estos medios para la gestión de trámites como por ejemplo, los pertenecientes a organismos públicos o la búsqueda de empleo. Por este motivo, el acercamiento de las TIC a grupos vulnerables como puede ser las personas con TMG favorece a su integración en la sociedad, pudiendo comportarse igual a cómo actúan el resto de la sociedad.

El hecho de que ciertas características de las TIC puedan influir en el desarrollo de delirios no debe ser una limitación para el acercamiento a estas tecnologías, sino un elemento a tener en cuenta por el terapeuta de cara a las evaluaciones e intervenciones (Nitzan et al., 2011).

Por otro lado, no se puede controlar la información presente en la red pero sí se puede mejorar la información aportada a los usuarios de Salud Mental así como darles la confianza para que acudan a ellos a la hora de resolver sus dudas. Además, para solventar barreras como el estigma o la falta de transporte y la accesibilidad a los servicios, podrían desarrollarse programas psicoeducativos online como el desarrollado por Soto-Pérez y Franco-Martín (2014).

Respecto a los beneficios y/o perjuicios que el uso de los grupos de ayuda online o las redes sociales tienen para las personas con TMG, éstos generalmente van a depender de las características individuales tales como la alfabetización digital, la cronicidad del trastorno, el objetivo con el que se accede a este medio, etc. Estos medios, al igual que para la población normal, pueden ser una vía para compartir información útil, comunicarse con otros y establecer o restablecer relaciones sociales. Estas nuevas experiencias interpersonales pueden favorecer a salir del aislamiento social que generalmente suelen tener y volver a recuperar una vida social normal. Pese a los beneficios, los profesionales han de conocer y seguir investigando cómo estas TIC debido a sus características pueden influir en algunos casos al empeoramiento o en la aparición de síntomas psicóticos.

\section{REFERENCIAS}

Abat, A., Pablo, S., Serrano, P., Fernández, E. y Martín, R. (2013). Necesidades de información y uso de internet en pacientes con cáncer de mama en España. Gaceta Sanitaria, 27, 241-247.

Akram, G., Boyter, A. y Thompson, A. (2010). Evaluation of antipsychotic information on UK schizophrenia websites. The Psychiatrist, 34, 422-426.

AmichaiHamburguer, Y. y Hayat, Z. (2011). The impact of the internet of the social lives of users: a representative sample from 13 countries. Computers in Human Behavior, 27, 585-589. 
Bach, P. y Hayes, S. C. (2002). The use of Acceptance and Commitment Therapy to prevent the rehospitalization on Psychotic Patients: A randomized controlled trial. Journal of Consulting and Clinical Psychology, 70, 1129-1139.

Berger, M., Wagner, T. H. y Baker, L. C. (2005). Internet use and stigmatized illness. Social Science and Medicine, 61, 1821-1827.

Buffardi, L. E. y Campbell, W. K. (2008). Narcissism and Social Networking Websites.Personality and Social Psychology Bulletin, 34, 1303-1324.

Cáceres, M. D., Ruíz, J. A. y Brändle, G. (2009). Comunicación interpersonal y vida cotidiana. La presentación de la identidad de los jóvenes en internet. Cuadernos de Información y Comunicación, 14, 213-231.

Catalano, G., Catalano, M. C., Embi, C. S., Frankel, R. I. (1999). Delusions about the internet.Southern Medical Journal, 92, 609-610.

Compton, M. T. (2003). Internet delusions.Southern Medical Journal, 96, 61-63.

Curtis, A. E. (2007). Theclaustrum: Sequestration of cyberspace. The Psychoanalytic Review, 1, 99-139.

Dixon, L. B., Lucksted, A., Medoff, D. R., Burland, J. y Stewart, B. (2011). Outcomes of a Randomized Study of a Peer-Taught Family-to-Family Education Program for Mental Illness. Psychiatric Services, 62, 591-597.

García, M. C. y Del Hoyo, M. (2013). Redes Sociales, un Medio para la Movilización Juvenil. ZER-Revista de Estudios de Comunicación, 18 (34), 111-125.

García Montes, J. M., Caballero Muñoz, D. y Pérez Álvarez, M. (2006). Changes in the self-resulting from the use of mobile phone. Media, Culture and Society, 28, 67-82.

Gaudiano, B. A. y Herbert, J. D. (2006). Acute treatment of in patients with psychotic symptoms using Acceptance and Commitment Therapy: pilot results. Behavior Research and Therapy, 44, 415-437.

Gergen, K. J. (1992). El yo saturado: dilemas de identidad en el mundo contemporáneo. Barcelona: Paidós.

Goldstein, M. (2002).Depression and anxiety in older women.Primary Care: Clinics in Office Practice, 29, 69-80.

Gonzalez, A. L. y Hancock, J. T. (2010). Mirror, Mirror on my Facebook Wall: Effects of Exposure to Facebook on Self-Esteem. Cyberpsychology, Behavior and Social Networking, 14, 79-83.

González, H., Pérez, M. (2007). La Invención de Trastornos Mentales. Madrid: Alianza Editorial.

Gorczynski, P., Patel H. y Ganguli, R. (2013). Evaluating the accuracy, quality, and readability of online physical activity, exercise, and sport information for people with schizophrenia.Mental Health and Physical Activity, 6, 95-99.

Gould, R. A., Mueser, K. T., Bolton, E., Mays, V. y Goff, D. (2001). Cognitive Therapy for psychosis in schizophrenia: An effect size analysis. Schizophrenia Research, 48, 335-342.

Greene, J. A., Choudhry, N. K., Kilabuk, E. y Shrank W. H. (2010). Online Social Networking by Patients with Diabetes: A Qualitative Evaluation of Communication with Facebook. Journal of General Internal Medicine, 26, 287-292. 
Guada, J. y Venable, V. (2011). A comprehensive Analysis of the Quality of Online Health-Related Information Regarding Schizophrenia.Health and Social Work, 36, 45-53.

Haker, H., Lauber, C. y Rössler, W. (2005). Internet forums: a self-help approach for individuals with schizophrenia? Acta Psychiatrica Scandinavica, 112, 474-477.

Houston, T. K., Cooper, L. A. y Ford, D. E. (2002). Internet support groups for depression: a 1-year prospective cohort study. American Journal of Psychiatry, 159, 2062-2068.

Instituto Nacional de Estadística (INE). (2014). Encuesta sobre Equipamiento y Uso de Tecnologías de Información y Comunicación en los Hogares: año 2014. Extraído el 3 de Enero de 2015 desde http://www.ine.es/prensa/np864. pdf.

Jiménez, J., García, J. F., Martín, J. L. y Bermúdez, C. (2007). Tendencias en el uso de internet como fuente de información sobre salud. UOC Papers, Revista sobre la sociedad del conocimiento, 4, 44-50.

Kaplan, K., Salzer, M. S., Solomon, P., Brusilovskiy, E. y Cousounis, P. (2011). Internet peer support for individuals with psychiatric disabilities: a randomized controlled trial. Social Science and Medicine, 72, 54-62.

Krishna, N., Fischer, A., Miller, M., Register-Brown, K., Patchan, K. y Hackman, A. (2013). The Role of social media networks in psychotic disorders: a case report. General Hospital Psychiatry, 35, 576-582.

Kummervold, P. E., Gammon, D., Bergvik, S., Johsen, J. A., Hasvold, T., Rosenvinge, J. H. (2002).Nordic Journal of Psychiatry, 56, 59-65.

Lieberman, J. A., Stroup, T. S., McEvoy, J. P., Swartz, M. S., Rosenheck, R. A., Perkins, D. O., Keefe, R., Davís, S. M., Davís, C. E., Lebowitz, B. D., Severe, J., Hsiaso, J. K. (2005). Effectiveness of Antipsychotic Drugs in Patients With Chronic Schizophrenia. The New England Journal of Medicine, 353, 1209-1223.

Mazanderani, F., O’Neill, B. y Powell, J. (2013). "People power" or “pester power"? Youtube as a forum for the generation of evidence and patient advocacy.Patient Education and Counseling, 93, 420-425.

Miller, B. J., Stewart, A., Schrimsher, J., Peeples, D. y Buckley, P. F. (2014). How connected are people with schizophrenia? Cell phone, computer, email, and social media use. Psychiatry Research, 12, 1-6.

Naslund, J. A., Grande, S. W., Aschbrenner, A. y Elwyn, G. (2014). Naturally occurring peer support through social media: The experiences of individuals with severe mental illness using Youtube. Plos One, 9 (10), 1-9.

Nitzan, U., Shosan, E., Lev-Ran, S. y Fennig, S. (2011). Internet-Related Psychosis- A Sign of the Times?The Israel Journal of Psychiatric and Related Sciences, 48, 207-211.

Perron, B. (2002). Online support for caregivers of people with a mental illness.

Psychiatric Rehabilitation Journal, 26, 70-77.

Read, J. (2008). Schizophrenia, drug companies and the internet.Social Science 
and Medicine, 66, 99-109.

Ritterband, L. M., Gonder-Frederick, L. A., Cox, D. J., Clifton, A. D., West, R. W. y Borowitz, S. M. (2003). Internet interventions: in review, in use, and into the future. Professional Psychology-Research and Practice, 34, 527-534.

Schranck, B., Sibitz, I., Unger, A. y Amering, M. (2010). How patients with schizophrenia use the internet: qualitative study. Journal of Medical Internet research, 12 (5), 70.

Soto-Pérez, F. y Franco-Martín, M. (2014).PsicoEd: una alternativa online y comunitaria para la psicoeducación en esquizofrenia. Psicoperspectivas, 13, 118-129.

Spinzy, Y., Nitzan, U., Becker, G., Bloch, Y. y Fennig, S. (2012). Does the internet offer social opportunities for individuals with schizophrenia? A crosssectional pilot study. Psychiatry Research, 198, 319-320.

Vázquez, M. B. y Martínez, R. S. (2011). Los Foros de Internet para Trastornos de Ansiedad y Depresión: un análisis comparativo del uso del lenguaje. Ansiedad y Estrés, 17, 15-25.

Veretilo, P. y Billick, S. B. (2012). Psychiatric Illness and Facebook: A Case Report. The Psychiatric Quarterly, 83, 385-389.

Vigod, S. N., Kurdyak, P. A., Dennis, C. L., Leszcz, T., Taylor, V. H., Blumbergerb, D. M. y Seitz, D. P. (2013). Transitional interventions to reduce early psychiatric readmissions in adults: Systematic review. The British Journal of Psychiatry, 202, 187-194.

Winzelberg, A. J., Classen, C., Alpers, G. W., Roberts, H., Koopman, C., Adams, R. E., Ernst, H., Dev, P. y Taylor, B. (2003). Evaluation of an internet support group for women with primary breast cancer.Cancer, 97, 1164-1173.

World Bank Institute (2008). Measuring Knowledge in the world's economies. Extraído el 3 de Enero de 2015 desde http://siteresources.worldbank.org/INTUNIKAM/Resources/KAM_v4.pdf

World Bank Institute (2015). Usuarios de internet por cada cien personas. Extraído el 2 de Enero de 2015. 